\title{
FLEXION AND SUPINATION DEFORMITIES OF THE ELBOW IN TETRAPLEGICS
}

\author{
By Alvin A. Freehafer, M.D. \\ Division of Orthopaedic Surgery, Highland View Hospital and Case Western Reserve School \\ of Medicine, Cleveland, Ohio, U.S.A.
}

Abstract. . Fixed flexion and supination deformities of the elbow occur occasionally in tetraplegics. The patients in whom this was seen were those who following injury had a neurological level at $\mathrm{C}_{5}$ and who subsequently developed radial wrist extension and brachioradialis function. They were generally patients who spent long hours with their elbows flexed and supinated.

A simple effective method of biceps tenotomy and plaster correction is described. Recurrence of deformity was seen if the flexion supination posture was continued. Strength of elbow flexion was not reduced. Patients maintained correction if they refrained from poor elbow posture and wore a simple plastic splint. The procedure of correction is not difficult and because of its simplicity it can be repeated if deformity recurs.

Key words: Flexion; supination deformity; elbow; tetraplegic; biceps; tenotomy.

MANY tetraplegics as a result of cervical spinal cord injury have good elbow flexion and supination with absence of triceps and pronator muscles. This muscle imbalance which tends to place the forearm in the flexed and supinated position occurs frequently but severe fixed deformity is rare.

Optimum use of the upper limb in the tetraplegic is dependent on absence of flexion and supination deformity of the forearm. Wheelchair push-ups and depressor transfers depend on full elbow extension to lock the elbow when triceps function is deficient. Severe flexion deformity makes propulsion of a wheelchair impossible. With the forearm pronated, the hand, by a variety of means, can develop prehensile function which is difficult when it is so deformed that the pronated position is impossible.

The purpose of this communication is to give our experience with flexion and supination deformities of the elbow, both of which tend to occur together and to discuss the incidence and management.

Mild deformities of the elbow can usually be improved by simple methods such as range of motion, exercises, practise with transfers and activities of daily living. Severe deformities with $45^{\circ}$ flexion contracture and inability to pronate beyond the neutral position are more difficult to correct. Because of the restrictions they place on the severely paralysed patient treatment and correction are important.

Zancolli (I968) has reported on transfer of the biceps tendon around to the radial side of the radius to allow for flexion and pronation. Occasionally a tight interosseous membrane requires release. This represents a rather extensive operative procedure which in our experience has not been necessary.

There are only eight patients who have developed a disabling flexion and supination deformity with 12 upper limbs involved. They all had severe paralysis initially with no muscle function below the biceps but subsequently over a period of I year gradually developed good radial wrist extensors and brachioradialis. All of these patients had done poorly in their efforts to become rehabilitated.

$$
15 / 3-c
$$


None was independent in wheelchair push-ups and transfers. They all were dismissed from the hospital with minimal deformity and returned months later with severe deformities. They all gave evidence of spending excessive periods of time in bed, lying supine with elbows flexed and supinated maximally.

\section{Case Reports}

Case No. I. R. M., a 2I-year-old man, was admitted to Highland View Hospital on I9 April 1976. He had been involved in a motorcycle accident I4 June I974 at which time he sustained a fracture dislocation of the fifth cervical vertebra on the sixth resulting in tetraplegia at the $\mathrm{C}_{5}$ level. He subsequently improved his function on the left so that at discharge he had good radial wrist extensors, brachioradialis, elbow flexion and supination and good shoulder control. Function on the right remained unchanged with no function below the biceps.
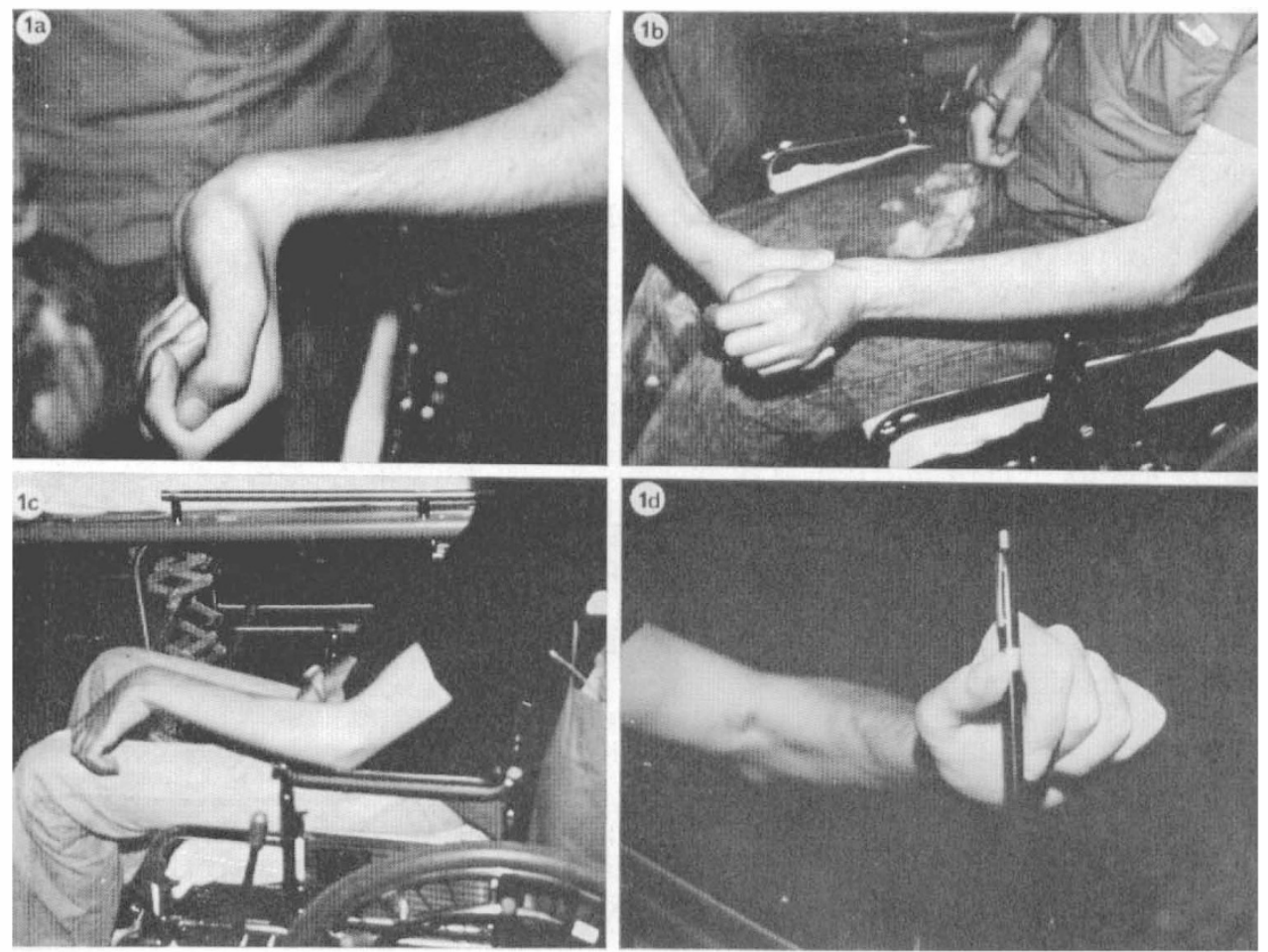

FIG. Ia

With normal relaxation and sitting the left hand falls into this awkward unusable position.

FIG. Ib

With passive extension and pronation the forearm is flexed $60^{\circ}$ and does not quite reach neutral rotation.

FIG. IC

Following treatment the left arm is nicely positioned with the hand in pronation.

FIG. Id

Key pinch demonstrated. 
The patient attempted college but dropped out after one semester and spent most of his time at home doing very little and making no plans for his future. At admission he had no deformity of the right upper limb but on the left he had a $60^{\circ}$ flexion deformity of the elbow and attempts at extending it caused maximum supination. One plaster cast was applied for I week in an attempt to gain maximum correction. Biceps tenotomy was done on 7 May 1976 followed by two more plaster casts. Full correction was attained. At the time of tenotomy the brachioradialis was transferred to give him thumb key pinch since he already had good automatic grasp. A plastic splint was used to maintain correction.

Case No. 2. R. K., a 29-year-old man, was admitted to Highland View Hospital on Io August I976. He was involved in a diving accident on 30 May I97I sustaining fracture dislocation of the fourth cervical vertebra on the fifth with initial paralysis at $\mathrm{C}_{5}$. He gradually developed good brachioradialis and radial wrist extensor function over a 6-month period. At discharge he had no deformity and was independent at wheelchair push-ups and transfers. He failed to return
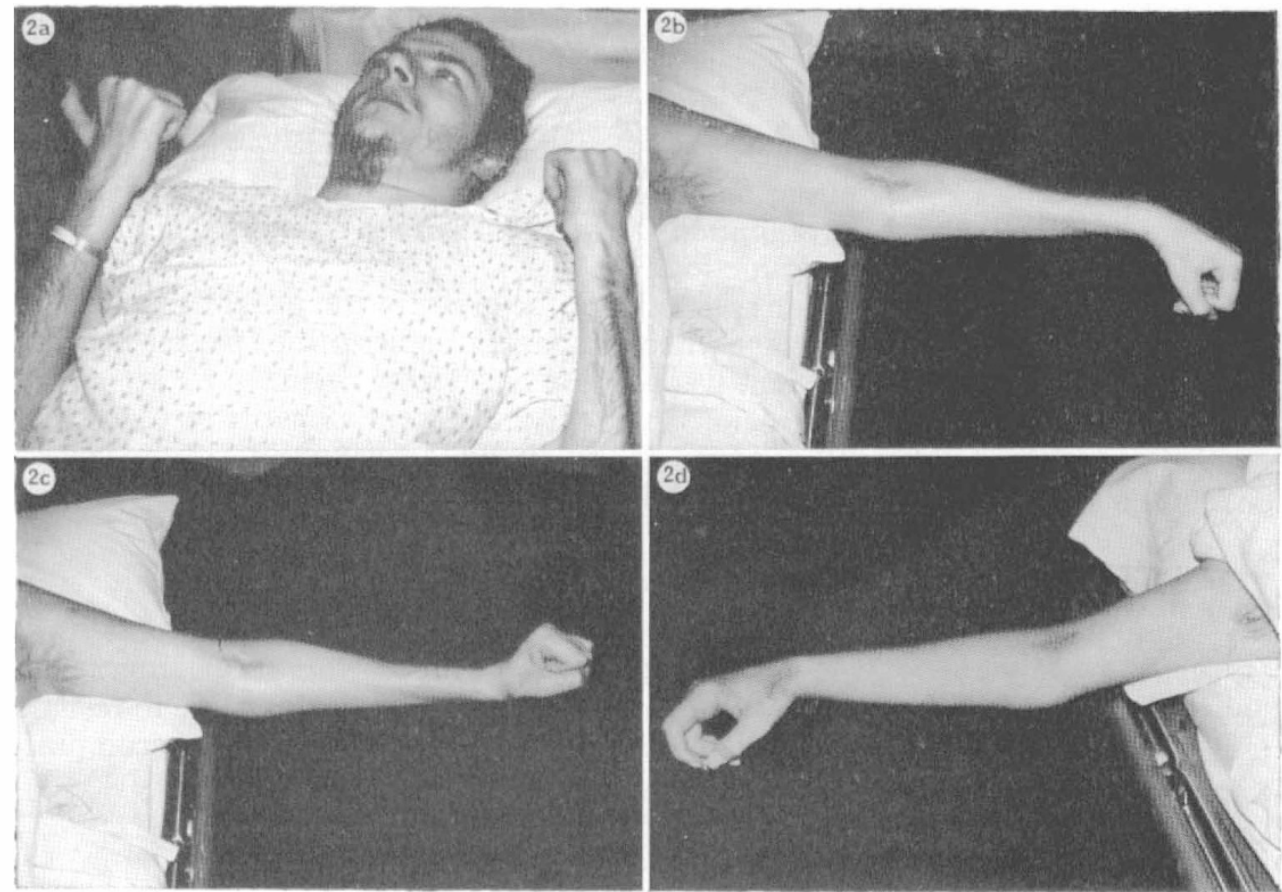

FIG. 2a

The posture of this patient which is so conducive to development of the flexion and supination deformities.

FIG. $2 b$

Full correction of the left elbow after treatment. Good automatic grasp is present.

FIG. 2c

Same left arm showing wrist flexed.

FIG. 2d

The right arm after correction showing pronation of forearm. 
for follow-up care until mid-1976 when he was found to have $90^{\circ}$ flexion contractures at both elbows and no pronation. Plaster casts changed weekly for 4 weeks corrected the deformities to $40^{\circ}$ with a few degrees of pronation. Biceps tenotomies were done on Io November 1976 and plaster casts were used at weekly intervals for 4 more weeks, fully correcting the deformities. Plastic splints were used to maintain correction. He subsequently learned to transfer again.

\section{Technique}

Well-padded long arm plaster casts were used and changed at weekly intervals. No force was used at any time. The upper limb was held in maximum extension and pronation as the plaster was applied. At the time plaster was changed it was remarkable how much correction occurred. When it was felt that no further correction could be obtained a biceps tenotomy was carried out through a $2 \mathrm{~cm}$ longitudinal incision at the distal arm over the tight tendon. The tendon and the lacertus fibrosa were cut and the wound closed. Plaster casts were continued and changed weekly until full correction was obtained.

Anaesthesia was used only for the tenotomy. Experience with other patients with head injuries or strokes with severe spasticity sometimes makes anaesthesia necessary for application of plaster casts but this was not needed in patients with spinal cord injury.

Roentgenograms of the elbows are mandatory to rule out heterotopic bone or other abnormalities contributing to flexion and supination deformities. Heterotopic bone has been the cause of limited elbow motion in the tetraplegic and should be removed to allow for better function.

A simple easily made plastic splint should be given to the patient to maintain correction. This is very important when the patient comes out of plaster because some discomfort makes the patient want to flex his elbows again.

Because many of these patients have abnormal sensation no force can be applied and casts must be well padded. If any discomfort or swelling of the fingers occurs the casts must be changed immediately.

\section{Results}

All patients maintained good position except two whose bilateral contractures redeveloped shortly after correction. This was probably because they continued to lie supine with their elbows flexed and supinated. One patient was able to resume transfers but the others never had the strength in their shoulders to accomplish this. No patient was made worse and no pressure sores occurred.

\section{Discussion}

It is of interest, that, in spite of deforming forces about the elbows seen in most of our tetraplegics so few develop severe flexion and supination deformities. This is probably because of their activities which call for extension at the elbow when they push up or transfer and because they need to use their hands with the forearms in pronation. It has been the tetraplegic who failed with rehabilitation who developed deformity. He then spent most of the time supine with forearms flexed and supinated. Once the deformity developed it tended to get worse.

Spasm may play a role in the development of deformity. The patients that were treated had increased reflexes but all had good voluntary control. Once the deformity was corrected spasm was not a problem. 
Manual muscle testing has not demonstrated any weakness of elbow flexion or supination. Admittedly, this is a crude test and some weakness must occur but probably less than occurs with transfer. All tendons healed in a lengthened position and seemed to function as well as they did prior to tenotomy. An alternative to tenotomy could well be lengthening of the tendon by Z-plasty but this was not done.

The deformities will surely recur if the patient reverts to old habits of lying supine and with elbows flexed and supinated. If activities are maintained at an optimum level the patients will be much improved and recurrences will not happen.

\section{SUMMARY}

Severe flexion and supination deformities of the forearm in tetraplegics occurs rarely. The absence of triceps function and pronation in a large number of these patients creates a muscle imbalance which tends to produce flexion and supination. The patient who is active and well rehabilitated has little difficulty, probably because he frequently uses his elbow in extension and his hand with the forearm pronated. It has been my observation that patients who lie for prolonged periods with the elbow flexed and supinated are the only ones who develop deformities which restrict their activities.

A simple method of correction has been described. Well padded plaster casts changed weekly or as indicated were used. Biceps tenotomy was done when no further correction could be obtained. This was followed by plaster casts changed weekly. At no time was force used. Plaster was changed if there was the slightest suggestion of discomfort or finger swelling.

\section{REFERENCE}

Zancolli, E. (1968). Structural and Dynamic Bases of Hand Surgery. J. B. Lippincott Company, Philadelphia. 